\title{
Variational Stereo Imaging of Oceanic Waves with Statistical Constraints
}

\author{
Guillermo Gallego, Anthony Yezzi, Francesco Fedele and Alvise Benetazzo
}

\begin{abstract}
An image processing observational technique for the stereoscopic reconstruction of the wave form of oceanic sea states is developed. The technique incorporates the enforcement of any given statistical wave law modeling the quasi Gaussianity of oceanic waves observed in nature. The problem is posed in a variational optimization framework, where the desired wave form is obtained as the minimizer of a cost functional that combines image observations, smoothness priors and a weak statistical constraint. The minimizer is obtained combining gradient descent and multigrid methods on the necessary optimality equations of the cost functional. Robust photometric error criteria and a spatial intensity compensation model are also developed to improve the performance of the presented image matching strategy. The weak statistical constraint is thoroughly evaluated in combination with other elements presented to reconstruct and enforce constraints on experimental stereo data, demonstrating the improvement in the estimation of the observed ocean surface.
\end{abstract}

Index Terms-Image processing, variational methods, stereo vision, multigrid, constrained statistical optimization, surface evolution, remote sensing, marine technology, wave measurements.

\section{INTRODUCTION}

V ISION-BASED remote sensing observational technology has gained popularity recently, specially in applications related to the measurement of ocean waves [1], [2], [3], [4], [5], [6], [7], [8]. Such topic is paramount to engineering because it enables the understanding of the space-time dynamics of ocean waves over an area rather than just at a point. This knowledge, contrasted with theoretical models, leads to better prediction of extreme events and renewed design of off-shore structures. Remote techniques based on image analysis alleviate some issues related to ocean wave measurements. Firstly, instrumentation is not directly in contact with sea water that is penalizing for hardware equipment. Secondly, image-based techniques provide wave data spatially distributed, allowing data analysis to be extended to the spacetime domain, rather than the classical time analysis for point probe data (e.g., from buoys or fixed wave meters). However,

G. Gallego was with the School of Electrical and Computer Engineering, Georgia Institute of Technology, Atlanta, Georgia 30332. He is now with the Grupo de Tratamiento de Imágenes, Universidad Politécnica de Madrid, 28040, Spain. E-mail: ggb@gti.ssr.upm.es

A. Yezzi is with the School of Electrical and Computer Engineering, Georgia Institute of Technology, Atlanta, Georgia 30332. E-mail: ayezzi@ece.gatech.edu

F. Fedele is with the School of Civil and Environmental Engineering and with the School of Electrical and Computer Engineering, Georgia Institute of Technology, Atlanta, Georgia 30332. E-mail: fedele@gatech.edu

A. Benetazzo is with Institute of Marine Science (ISMAR), National Research Council (CNR), 30122 Venice, Italy. E-mail: alvise.benetazzo@ve.ismar.cnr.it more processing power is required to reveal the desired physical information from the observed video data. Now, with increasingly more computing power, memory resources and better optical instrumentation, many image processing systems are being developed to study our planet [2]-[8]. The Wave Acquisition Stereo System (WASS) [2] and the trinocular imaging system (ATSIS) [3], [5] are successful examples that apply epipolar techniques to show that the accuracy of such image processing systems is comparable to the accuracy obtained from traditional instrumentation. More recently, an application of stereo data to estimate the space-time statistics of sea waves can be found in [9].

The problem of the stereoscopic reconstruction of the surface of the ocean has some particular characteristics. Since the object of interest lacks photometrically distinct "features" due to its weakly textured surface, traditional image-based stereo methods [10, Ch.11] may fail to provide a satisfactory reconstruction. In favorable conditions and often using a postprocessing hole-filling strategy, such methods provide a sparse reconstruction [2], [3]. In contrast, modern object-based stereo methods [11], [12] founded upon variational optimization and partial differential equations (PDE) are more robust to image matching problems and provide dense and continuous surface reconstructions. So far, both traditional and variational stereo techniques do not include in the reconstruction process prior information of the physics of ocean waves; this is usually verified a posteriori [4], [8]. The oceanographic community has developed statistical and spectral models for the characterization of sea states [13], [14], [15], [16] clearly indicating that oceanic waves are quasi-Gaussian in nature and it would be desirable to enforce such physical information during the reconstruction process.

Here we develop a method that combines variational stereo techniques with statistical models of ocean waves to recover the shape of the surface of the ocean. Although the method is applied to an ocean engineering problem, it is of interest to other research areas since the idea of incorporating global constraints, such as statistical models, within a variational estimation process is an appealing problem solving approach.

This work is a more fully developed version of the conference paper [17]. Stemming from the method in [18], a statistical distribution is enforced as a prior in the stereo reconstruction process via a weak constraint in the variational optimization framework. With respect to [17], two new measures of statistical dissimilarity (Anderson-Darling and Cramér von Mises) are discussed and all (four) measures of statistical dissimilarity are presented in a unified methodology. The technique in [17], [18] is improved by augmenting the 
photometric model with automatic gain control and spatial intensity compensation parameters to alleviate variations in illumination. A logarithmic intensity transformation is also used to improve the robustness and performance of the presented image matching strategy. The structure of the paper emphasizes the simultaneous optimization of both the surface parameters as well as the new image compensation parameters. The experimental section has been significantly expanded with respect to [17] to test the new parameters of the photometric criterion and all four weak statistical constraint penalties (previously, only the "CDF" penalty was evaluated). In addition, the technique is demonstrated on the simultaneous estimation of several snapshots of a stereo video. Final remarks and future research directions conclude the paper.

\section{The VARiational FramewORK}

\section{A. Multi-image Setup and Graph Surface Representation}

Let us consider a scenario with a set of $N_{c}$ calibrated cameras $\mathrm{P}^{i}, i=1, \ldots, N_{c}$, taking images $I_{i}$ of an object whose (smooth) surface is $S \subset \mathbb{R}^{3}$ with local coordinates $(u, v) \in \mathbb{R}^{2}$. A camera is modeled as an ideal perspective projection [19] $\pi_{i}: \mathbb{R}^{3} \rightarrow \mathbb{R}^{2}$ mapping a surface point (or, in general, a 3-D point) $\mathbf{X}=(X, Y, Z)^{\top}$ to point $\mathbf{x}_{i}=\pi_{i}(\mathbf{X})$ in the $i$-th image, where $\mathbf{x}_{i}=\left(x_{i}, y_{i}\right)^{\top}$. This mapping is linear if homogeneous coordinates are used: $\overline{\mathbf{x}}_{i} \sim \mathrm{P}^{i} \overline{\mathbf{X}}$, where $\overline{\mathbf{x}}_{i}=\left(\mathbf{x}_{i}^{\top}, 1\right)^{\top}, \overline{\mathbf{X}}=\left(\mathbf{X}^{\top}, 1\right)^{\top}$ and symbol $\sim$ means equality up to a nonzero scale factor. The $3 \times 4$ projection matrix $\mathrm{P}^{i}=\mathrm{K}^{i}\left[\mathrm{R}^{i} \mid \mathbf{t}^{i}\right]$ consists of the intrinsic $\left(\mathrm{K}^{i}\right)$ and extrinsic $\left(\mathrm{R}^{i}, \mathrm{t}^{i}\right)$ calibration parameters of the $i$-th camera. The optical center of the $i$-th camera is point $\mathbf{C}_{i}=\left(C_{i}^{1}, C_{i}^{2}, C_{i}^{3}\right)^{\top}$ satisfying $\mathrm{P}^{i} \overline{\mathbf{C}}_{i}=\mathbf{0}$. Finally, let $I_{i}\left(\mathbf{x}_{i}\right)$ be the image intensity at $\mathbf{x}_{i}$. Fig. 1 shows the geometry of the scene under study.

Assume that the surface of the object can be represented in the form of a graph or elevation map,

$$
Z=Z(X, Y),
$$

where $Z$ is the height of the surface with respect to a parametrizing plane with coordinates $X, Y$. Slow varying, nonbreaking waves can be represented in this form with respect to a plane orthogonal to gravity direction. The graph representation of the water surface exhibits some clear advantages over the more general level set representation of active surfaces in [11], [4]. The surface is estimated directly using the height function (1) discretized over a fixed 2-D grid defined on the $X Y$ plane, instead of using a more expensive 3-D grid for the level set function. Higher spatial resolution (finer details) can be achieved with the graph representation than with the level set. The graph representation simplifies both the geometric description of the surface and its photometric characterization. It also allows for a broader class of numerical solvers besides gradient descent, such as Fast Poisson Solvers, Multigrid Methods, Finite-Element Methods, etc.

However, to use the graph representation, a world frame properly oriented with the gravity direction must be defined in advance, which is not trivial. Also, a constrained estimation process (forcing the surface to remain in the form of a graph) may be slower than an unconstrained one.

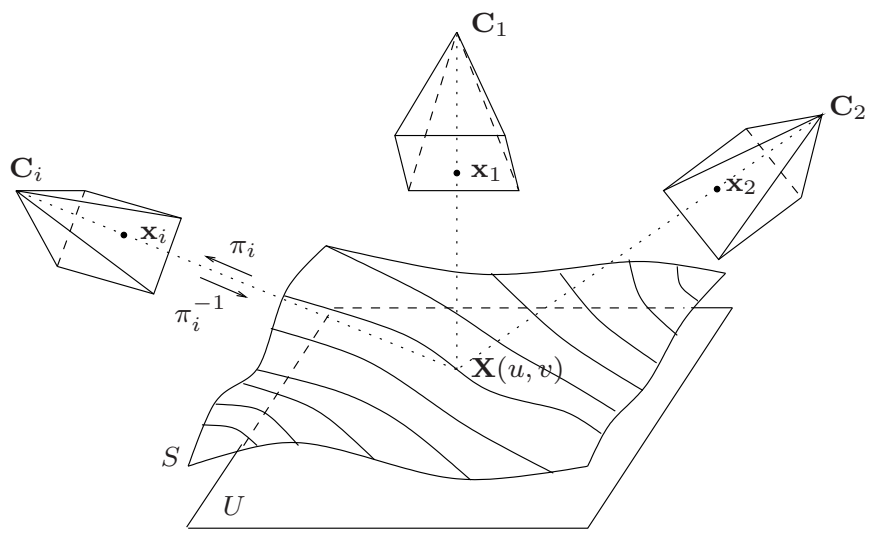

Fig. 1. Scene: camera setup and surface $S$ in the form of a graph. Cameras are represented by quadrilateral pyramids whose apexes are the optical centers $\mathbf{C}_{i}$ and whose bases represent the image planes $\Omega_{i}$ where intensities $I_{i}\left(\mathbf{x}_{i}\right)$ are recorded. A surface point has coordinates $\mathbf{X}(u, v)=(u, v, Z(u, v))^{\top}$. $\pi_{i}^{-1}$ denotes the back-projection operation from a point in the $i$-th image to the closest surface point with respect to the camera (see Appendix A).

\section{B. Generative Model and Variational Approach}

To infer the 3-D shape of the surface $S$ from a collection of $N_{c} \geq 2$ images $I_{i}$, we investigate a generative model of the scene. Instead of using a back-projection model typical of sparse feature-based 3-D reconstructions, we use a forward image formation model and measure the discrepancy between the modeled images $\hat{I}_{i}$ generated by a candidate surface and the actual observations $I_{i}$. Then, we seek for the candidate surface that minimizes the cost functional quantifying such discrepancy. Our model implies the joint estimation of the shape of the surface $S$ and the radiance function $f$ on the surface needed to compute the modeled images. Let the data fidelity cost $E_{\text {data }}(S, f, \vartheta)$ measure the photo-consistency of the model with respect to the observations:

$$
E_{\text {data }}:=\sum_{i=1}^{N_{c}} E_{i}, \quad E_{i}:=\int_{\Omega_{i}} \phi_{i} d \mathbf{x}_{i}
$$

where a possible photometric matching criterion is

$$
\phi_{i}:=\frac{1}{2}\left(I_{i}\left(\mathbf{x}_{i}\right)-\hat{I}_{i}\left(\mathbf{x}_{i}, f, \boldsymbol{\vartheta}_{i}\right)\right)^{2} .
$$

The region of the image domain where the surface is projected is denoted by $\Omega_{i}$. Both intensities in (3) for a candidate surface $(S, f)$ are detailed in Appendix A. The modeled intensity $\hat{I}_{i}\left(\mathbf{x}_{i}, f, \boldsymbol{\vartheta}_{i}\right)$ may include automatic gain control and spatial intensity compensation (SIC) components $\boldsymbol{\vartheta}_{i}:=\left(a_{i}, \boldsymbol{\theta}_{i}^{\top}\right)^{\top}$ to account for systematic mismatched intensities due to different camera gain settings, vignetting, direction of sunlight illumination, etc. For clarity purposes, this model is explained in Appendix A. It suffices to say that there is a SIC parameter vector $\boldsymbol{\vartheta}:=\left(\boldsymbol{\vartheta}_{1}^{\top}, \ldots, \boldsymbol{\vartheta}_{N_{c}}^{\top}\right)^{\top}$ with $\left(N_{s}+1\right)$ elements per image, that affects the photometric fit of the model to the observed images and that this vector has to be estimated for an optimal fit.

An attempt to minimize (2) will produce a non-smooth surface and radiance. Therefore, to have a well-posed variational problem, let us minimize a sum of the data fidelity term (2) and two regularizing terms in the geometry $\left(E_{\text {geom }}\right)$ and in the 
radiance $\left(E_{\mathrm{rad}}\right)$ of the surface,

$$
E(S, f, \boldsymbol{\vartheta})=E_{\text {data }}(S, f, \boldsymbol{\vartheta})+\alpha E_{\text {geom }}(S)+\beta E_{\text {rad }}(f),
$$

where $\alpha, \beta \in \mathbb{R}^{+}$. Motivated by the common parametrizing domain of the shape and radiance of the surface and to obtain the simplest diffusive terms in the necessary optimality conditions of the cost (4), let us choose

$$
\begin{aligned}
E_{\text {geom }} & :=\frac{1}{A} \int_{U} \frac{1}{2}\|\nabla Z(\mathbf{u})\|^{2} d \mathbf{u}, \\
E_{\mathrm{rad}} & :=\frac{1}{A} \int_{U} \frac{1}{2}\|\nabla f(\mathbf{u})\|^{2} d \mathbf{u},
\end{aligned}
$$

where $A=\int_{U} d \mathbf{u}$ is the area of the (fixed) domain of integration $U$ (the part of the parameter space whose surface projects on $\Omega_{i}$ in the $i$-th image), $\nabla Z(\mathbf{u})=\left(Z_{u}, Z_{v}\right)^{\top}$, $\nabla f(\mathbf{u})=\left(f_{u}, f_{v}\right)^{\top}$ and subscripts indicate the derivative with respect to that variable. Since the normalization factor $A$ in (5) and (6) is constant, it can be subsumed in coefficients $\alpha$ and $\beta$.

The cost $E_{i}$ in (2) integrates a point-wise comparison of the observed and modeled intensities at $\mathbf{x}_{i} \in \Omega_{i}$. Apparently, this is an ineffective criterion compared to a neighborhood-wise matching of the intensities, however the regularizers $E_{\text {geom }}$ and $E_{\text {rad }}$ account for such neighborhood effects, extending the local influence of a point-wise photometric criterion.

Two main advantages arise from defining the data fidelity cost as an integral over the image domain (rather than over the parameter space $U):(i)$ the data term is independent of the choice of parametrizing plane for the graph, and (ii) the resulting optimality conditions for the minimization of (4) depend only upon image values $I_{i}$, not the image derivatives $\nabla I_{i}$; these are transferred to the radiance model, $\nabla f$. Derivatives of the radiance model are favorable from a numerical standpoint compared with derivatives on the image since the radiance model (by the regularizing constraint $\left.E_{\text {rad }}\right)$ is smoother than the image. This desirable property is inherited from the modeling and mathematical principles that we follow from [12]. The resulting algorithm is more robust to image noise than other variational approaches for stereo 3-D reconstruction.

Once all terms in (4) have been specified, they are expressed in the same domain by rewriting (2) as

$$
E_{i}=\int_{\Omega_{i}} \phi_{i} d \mathbf{x}_{i}=\int_{U} \phi_{i} \mathrm{~J}_{i} d \mathbf{u},
$$

where $\mathrm{J}_{i}$ is the Jacobian of the change of variables between integration domains $\Omega_{i}$ and $U$ (see Appendix B). After collecting terms (4) and (7), and noting that the surface shape solely depends on the height (by (23)), the cost becomes

$$
E(Z, f, \boldsymbol{\vartheta})=\int_{U} L(Z, \nabla Z, f, \nabla f, u, v, \boldsymbol{\vartheta}) d \mathbf{u} .
$$

where the integrand is the so-called Lagrangian.

\section{Weak Enforcement of Elevation Distributions}

One further advantage of our variational generative model over image-based stereo methods is that it allows to include properties of the physics of the waves in the model. For example, we may account for global statistical properties by weakly enforcing them using an extra cost in the functional. Such cost penalizes the discrepancy between the statistics of the reconstructed surface and those consistent with a physical model. In particular, we may penalize the deviation of the height distribution of the water surface with respect to a physically-justified Gaussian model and drive the surface evolution toward (weakly) satisfying such a global property.

If $Z(\mathbf{u})=Z(u, v)$, with $\mathbf{u}=(u, v)^{\top}$, is the height of the surface (wave) and it is interpreted as a random variable, then its cumulative distribution function $(\mathrm{CDF})$ is

$$
\operatorname{cdf}^{Z}(z):=P(Z \leq z)=\frac{1}{A} \int_{U} H(z-Z(\mathbf{u})) d \mathbf{u},
$$

where $H(\cdot)$ is the Heaviside function. Accordingly, the probability density function (PDF) is, in the distributional sense,

$$
\operatorname{pdf}^{Z}(z):=\frac{d}{d z} \operatorname{cdf}^{Z}(z)=\frac{1}{A} \int_{U} \delta(z-Z(\mathbf{u})) d \mathbf{u},
$$

where $\delta(\cdot)$ is the Dirac delta function.

Suppose (4) is augmented with a cost $\gamma E_{\mathrm{cdf}}(S), \gamma \geq 0$, that measures the discrepancy between a target height CDF that we wish to enforce, $G$, and the experimental CDF of the height. A sensible measure compares the standardized (centered and normalized), CDFs in the $\mathrm{Z}$-score variable $\xi=\left(Z-\mu_{Z}\right) / \sigma_{Z}$ (assuming $\left.\sigma_{Z} \neq 0\right), \operatorname{cdf}^{\xi}(x)=\operatorname{cdf}^{Z}\left(\mu_{Z}+\sigma_{Z} x\right)$ and $G_{n}(x)=$ $G\left(\mu_{Z}+\sigma_{Z} x\right)$ via

$$
\widetilde{E}_{\mathrm{cdf}}(Z):=\frac{1}{2}\left\|G_{n}-\mathrm{cdf}^{\xi}\right\|_{L^{2}}^{2},
$$

where $\|g\|_{L^{2}}^{2}=\langle g, g\rangle_{L^{2}}$ is the usual squared norm in the Lebesgue space $L^{2}(\mathbb{R})$, with inner product $\langle f, g\rangle_{L^{2}}=$ $\int_{-\infty}^{\infty} f(x) \overline{g(x)} d x$, so that the same penalty is obtained regardless of the amplitude and average of the waves. Cost (9) is expressible in terms of the original (unstandardized) height CDFs by

$$
\widetilde{E}_{\mathrm{cdf}}=\sigma_{Z}^{-1} E_{\mathrm{cdf}}
$$

where $E_{\text {cdf }}:=\frac{1}{2}\left\|G-\operatorname{cdf}^{Z}\right\|_{L^{2}}^{2}$. If the factor $\sigma_{Z}^{-1}$ is approximately constant, it can be subsumed in $\gamma$. Otherwise, it should be considered in the optimization of the composite cost with respect to the height. In this work, we assume that $\sigma_{Z}$ is approximately constant since the statistical penalty is used to refine a solution that approximately minimizes the other terms in the proposed cost.

Theoretical probabilistic models that can be used as target physical elevation distributions $G$ are given in [15], [16]. These models are quasi-Gaussian distributions that capture the asymmetry present in real life water waves, which have steep crests and shallow troughs.

\section{Functional Minimization. Optimality Condition}

The composite cost functional

$E(Z, f, \boldsymbol{\vartheta})=E_{\text {data }}(Z, f, \boldsymbol{\vartheta})+\alpha E_{\text {geom }}(Z)+\beta E_{\text {rad }}(f)+\gamma \widetilde{E}_{\text {cdf }}(Z)$

depends on two functions (the shape $Z$ and the radiance $f$ of the surface) and a finite number of parameters $\vartheta$. To find a minimizer of such a functional, we derive the necessary 
optimality condition by setting to zero the first variation of the functional, yielding a system of equations:

$$
\begin{aligned}
& \frac{\delta E}{\delta Z}=0=\frac{\delta E}{\delta f}, \\
& \frac{\partial E}{\partial \boldsymbol{\vartheta}}=\mathbf{0} .
\end{aligned}
$$

Equations (11), where $\delta E / \delta w$ stands for the functional derivative of $E$ with respect to $w$, can be further written as

$$
\begin{aligned}
g(Z, f, \boldsymbol{\vartheta})-\bar{\alpha} \Delta Z & =0 & & \text { in } U, \\
b(Z, f, \boldsymbol{\vartheta})+\bar{\alpha} \frac{\partial Z}{\partial \boldsymbol{\nu}} & =0 & & \text { on } \partial U, \\
-\sum_{i=1}^{N_{c}} a_{i}\left(I_{i}-\hat{I}_{i}(f, \boldsymbol{\vartheta})\right) \mathrm{J}_{i}(Z)-\bar{\beta} \Delta f & =0 & & \text { in } U, \\
\bar{\beta} \frac{\partial f}{\partial \boldsymbol{\nu}} & =0 & & \text { on } \partial U,
\end{aligned}
$$

where $\bar{\alpha}=\alpha / A$ and $\bar{\beta}=\beta / A$. Equations (13)-(16) form a coupled system of PDEs (EL equations) along with boundary conditions. The derivation can be found in [18] without the SIC model and the terms corresponding to the statistical constraint. The Laplacians $\Delta Z$ and $\Delta f$ arise from the regularizing terms (5) and (6), and $\partial \cdot / \partial \nu$ is the the directional derivative along $\boldsymbol{\nu}=\left(\nu^{u}, \nu^{v}\right)^{\top}$, the normal to the integration domain $U$. The optimality with respect to the SIC parameters (12) is derived in Appendix C.

The non-linear term $g(Z, f, \boldsymbol{\vartheta})$ in (13) is the weighted sum of two terms: one due to the data fidelity cost,

$$
g_{\text {data }}(Z, f, \boldsymbol{\vartheta})=\nabla f \cdot \sum_{i=1}^{N_{c}} \frac{a_{i}\left|\mathrm{M}^{i}\right|}{\tilde{Z}_{i}^{3}}\left(I_{i}-\hat{I}_{i}\right)\left(u-C_{i}^{1}, v-C_{i}^{2}\right),
$$

which combines the geometric and photometric elements of the scene (see Appendices A and B), and one due to the statistical penalty,

$$
g_{\mathrm{cdf}}(Z)=\left.\frac{1}{A}\left(G(z)-\operatorname{cdf}^{Z}(z)\right)\right|_{z=Z(\mathbf{u})} .
$$

Calculations are given in Appendix D, and, in case of varying $\sigma_{Z}$, the calculations are given in Appendix E.

Each of the PDEs in the coupled system of equations (13)(16) can be characterized as follows. For a fixed shape, (15) and (16) form a linear elliptic PDE with Neumann boundary conditions, whereas, for a fixed radiance, (13) and (14) form a nonlinear elliptic PDE in the height $Z$ with nonstandard boundary conditions. Assuming that the data fidelity term vanishes close to the boundary allows us to approximate (14) by a homogeneous Neumann boundary condition. This is a reasonable hypothesis since the major contribution to the cost is given by the terms inside $U$.

To solve difficult EL equations such as the ones considered here it is standard to use the gradient descent method, which guides the unknowns through a path of decreasing cost to reach a local minimum of the functional. Such solutions are characterized by stable steady states of augmented PDEs in artificial time $t$. This is the context of the so-called active surfaces in vision, where the solution is obtained by deforming an initial surface via the gradient flow given by the EL equations of the
TABLE I

CDF INNER PRODUCT WEIGHT FUNCTIONS IN EQ. (21) FOR DIFFERENT ERROR CRITERIA. $G(z)$ AND $G^{\prime}(z)$ DENOTE THE CDF AND PDF OF THE TARGET DISTRIBUTION, RESPECTIVELY.

\begin{tabular}{|l|c|}
\hline Error criterion & Weight function $w(z)$ \\
\hline \hline Anderson - Darling (AD) & $G^{\prime}(z) /(G(z)(1-G(z)))$ \\
\hline CDF & 1 \\
\hline Cramér - von Mises (CvM) & $G^{\prime}(z)$ \\
\hline
\end{tabular}

cost functional. Due to the asymmetry in the complexity of the equations, a minimization strategy consisting of a nested iterative scheme is proposed: an outer loop performs a gradient descent in the height $Z$ and an inner loop implements a direct optimization for the radiance and the SIC parameters $(f, \boldsymbol{\vartheta})$. The latter is possible since (11)-(12) can be solved for the optimal $\vartheta^{*}$ and $f^{*}$ in terms of $Z$.

\section{E. Other Measures of Statistical Dissimilarity}

Another reasonable score to measure the statistical discrepancy between the empirical distribution of the wave field and the one dictated by the physical model is the $L^{2}$ difference between standardized PDFs:

$$
\widetilde{E}_{\mathrm{pdf}}(Z):=\frac{1}{2}\left\|G_{n}^{\prime}-\mathrm{pdf}^{\xi}\right\|_{L^{2}}^{2},
$$

where $G_{n}^{\prime}(x)=\sigma_{Z} G^{\prime}\left(\mu_{Z}+\sigma_{Z} x\right)$ is the target PDF that we wish to enforce and $\operatorname{pdf}^{\xi}(x)=\sigma_{Z} \operatorname{pdf}^{Z}\left(\mu_{Z}+\sigma_{Z} x\right)$. These relations preserve the unit area property of PDFs. The prime notation ' designates the derivative. If $E_{\mathrm{pdf}}:=$ $\frac{1}{2}\left\|G^{\prime}-\mathrm{pdf}^{Z}\right\|_{L^{2}}^{2}$, then $\widetilde{E}_{\mathrm{pdf}}=\sigma_{Z} E_{\mathrm{pdf}}$. Penalty (19) leads to a non-linear contribution to $g$ in (13) of the form (see Appendix D)

$$
g_{\mathrm{pdf}}(Z)=-\left.\frac{1}{A} \frac{d}{d z}\left(G^{\prime}(z)-\operatorname{pdf}^{Z}(z)\right)\right|_{z=Z(\mathbf{u})} .
$$

Enforcing the statistical constraint via the $L^{2}$ difference of characteristic functions (i.e. the Fourier transform of the PDFs) is, by Parseval's theorem, equivalent to the above PDF approach. Details can be found in [20].

The Anderson-Darling (AD) test [21] and the Cramér-von Mises ( $\mathrm{CvM}$ ) criterion inspire other judicious expressions to asses the statistical dissimilarity, adopting the form of weighted norms in $L^{2}(\mathbb{R})$,

$$
E_{\text {stat }}:=\int_{-\infty}^{\infty} w(z) \frac{1}{2}\left(G(z)-\operatorname{cdf}^{Z}(z)\right)^{2} d z,
$$

Table I collects some choices of the weight function and Fig. 2 shows them for a Gaussian distribution. The AD weight function places more influence on observations in the tails of the distribution, whereas the CvM weight emphasizes the observations in the central part, i.e., around the mean. The CDF approach (9) lies between them, weighting all observations equally. Penalty (21) implies a non-linear contribution to $g$ in (13) of the form

$$
g_{\text {stat }}(Z)=w(Z(\mathbf{u})) g_{\text {cdf }}(Z) .
$$

The proof is analogous to that of (18) in Appendix D. In the same spirit, criterion (19) can be extended to compare PDFs using weighted norms in $L^{2}(\mathbb{R})$. 


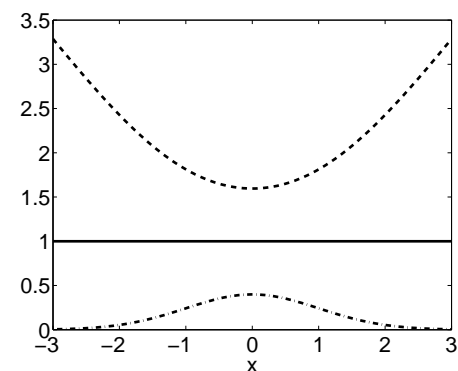

Fig. 2. Weight functions of Table I corresponding to a Gaussian distribution From top top bottom: Anderson-Darling (AD) weight, unit weight and Cramér-von Mises (CvM) weight.

\section{F. Improved Robustness of the Model}

There are some situations where the performance of the variational model may deteriorate. For example, the model is designed for scenes with objects whose surfaces obey the Lambertian light reflection hypothesis, but in practice, images of ocean waves acquired under favorable circumstances only follow this rule approximately and therefore, the reconstruction of the waves worsens as the reflectance of the scene departs from the Lambertian case. In addition, breaking waves generate localized white regions on the water surface that contrast with the darker neighboring intensities. According to the proposed data fidelity cost (2)-(3), a mismatch in the reconstruction of these regions contributes more to the total cost (in a quadratic manner) than mismatched regions with smaller intensity jumps. Therefore, to minimize the composite cost, the descent flow that drives the surface shape evolution concentrates its efforts in reducing the error in these regions, generating localized high frequencies that are not present in the physical surface of the waves. Thus, in these regions, the model generates undesired artifacts.

Several approaches have been investigated in [20] to address both aforementioned conflictive situations. One approach consists of modifying the photometric criterion (3) using subquadratic comparison functions so that errors are weighted differently according to their value and large errors do not capture the attention of the minimizing flow. This same idea of weighting the residuals differently across the scale is closely related to the theory of robust M-estimators in statistics. However, this approach increases the amount of non-linearity in the model and in the EL equations. The advantage of the quadratic model (3) is that it produces a linear radiance PDE (15), linear equations for the SIC parameters (12) (see Appendix C), and mild non-linearities in the height PDE (17).

A better approach to attenuate the effect of specularities and sea foam on the reconstruction algorithm consists of using the quadratic error function (3) acting on a logarithmic version of the images. This has the effect of stretching dark grayscale intensity levels and compressing white intensity levels. The latter is of most significance to our ocean wave application because it compresses large intensity jumps near white regions caused by specularities and sea foam. For images with intensity levels in the range $[0,255]$, the transformation rule is given by $y(x)=255 \log (1+c x) / \log (1+255 c)$, where $x$ and $y$ are the input and output intensities. The parameter
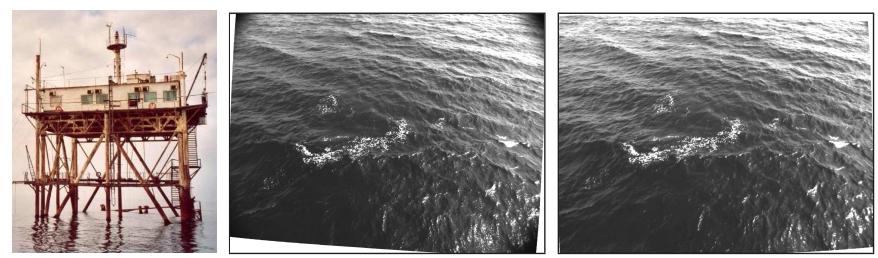

Fig. 3. Images (middle and right) acquired by two cameras mounted on an offshore platform (left)

$c>0$ controls the amount of stretching and compression of the intensity levels. In practice, it has been observed that the choice $c=1$ yields good results. The relationship between the weights of the different terms in the composite cost functional is affected by the logarithmic transformation.

\section{G. Numerical Solution.}

The optimality equations (11) and (12) are discretized on a rectangular 2-D grid in the parameter space, and finite differences are used to approximate the derivatives. Following the strategy of the nested iterative solver, the optimization of the SIC parameters implies the solution of a linear system of equations (12), as explained in Appendix C, whereas direct optimization of the radiance is achieved using stationary iterative methods (Jacobi or Gauss-Seidel). The gradient descent PDE for the height is numerically approximated by an explicit updating scheme using forward differences in time and central differences in space. The von Neumann stability analysis of the linearized PDE yields a time step $\Delta t \leq 1 /\left(\frac{4 \alpha}{h^{2}}+\frac{1}{2} \max \left|g^{\prime}(Z)\right|\right)$, where $g^{\prime}$ is the derivative of $g$ in (13) and the maximum is taken over the 2-D discretized grid at current time $t$. The time step may change at every iteration. The logarithmic pre-processing of the image intensities has one further advantage: it decreases the value of $\max \left|g^{\prime}(Z)\right|$, therefore increasing the time step and the convergence rate of the iterative solver. If $\gamma>0$, an additional constraint is required on the time step: the maximum height increment must be of the order of the bin size used to estimate the experimental $\mathrm{CDF} / \mathrm{PDF}$ so that each iteration does not drastically change the statistics of the surface height.

Both updating schemes (stationary methods for $f$ and the time-stepping method for $Z$ ) are used as relaxation procedures inside a multigrid method [22] that approximately solves the EL equations. Multigrid methods are the most efficient numerical tools for solving elliptic boundary value problems.

\section{EXPERIMENTS}

The variational method is applied to a pair of stereo images acquired at an off-shore platform near the southern seashore of the Crimean peninsula, in the Black Sea. The water depth at the platform is $30 \mathrm{~m}$. Two cameras mounted $12 \mathrm{~m}$ above the mean sea level and with a baseline of 2.5 meters acquire images of size $1624 \times 1236$ pixels (see Fig. 3). During acquisition, there were swell and developing waves with spectral peaks at about $0.2 \mathrm{~Hz}$ and $0.4 \mathrm{~Hz}$, respectively. The wind speed measured at $23 \mathrm{~m}$ height was around $8 \mathrm{~m} / \mathrm{s}$. 

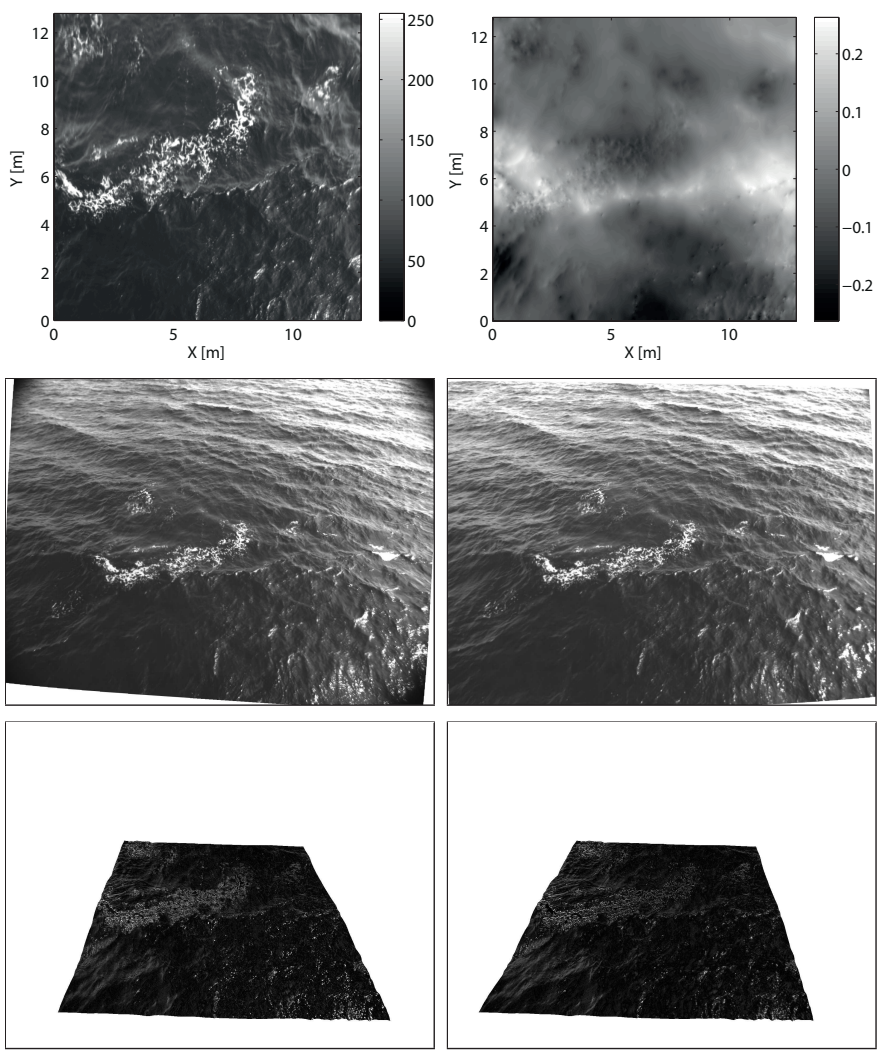

Fig. 4. Surface reconstruction without statistical constraint. Top: radiance and height functions, $f(\mathbf{u})$ and $Z(\mathbf{u})$, after running FMG method without statistical constraint $(\gamma=0)$. Height function $Z(\mathbf{u})$ is represented by grayscale intensities, from dark (low) to white (high). Middle: modeled images $\hat{I}_{i}, i=1,2$, superimposed on original images. Bottom: corresponding absolute error images $\left|I_{i}-\hat{I}_{i}\right|$ within the projection domains $\Omega_{i}$; error intensities have been amplified by a factor of 10 for visualization.

The graph of the surface is discretized on a grid with $N_{p}=513^{2}$ points and resolution $h=2.5 \mathrm{~cm}$, thus covering an area of $13 \times 13 \mathrm{~m}^{2}$. Approximately, 1 pixel corresponds to a physical displacement of $1.06 \mathrm{~cm}(1.88 \mathrm{~cm})$ for grid points near (resp. far from) the cameras. Both displacements are of the same order as $h$. Processing is performed in a notebook computer with an Intel $\AA$ Core $^{\mathrm{TM}}$ i5 $560 \mathrm{M}(2.66 \mathrm{GHz})$ CPU. The multi-core implementation is done in non-optimized C++ code with OpenMP $\AA$ (Open Multi-Processing), the de-facto standard that implements multithreading for parallel programming on shared memory systems.

\section{A. Effect of the Statistical Constraint}

The linearized optimality PDEs are solved iteratively until a local solution is reached by means of a 6-level full multigrid method [22] with 400 iterations per level, 2 V-cycles per iteration, and 1 pre- and post-relaxation sweeps per cycle. The weights of the regularizers are set to $\alpha / A=0.1$ and $\beta / A=0.025$. This processing stage takes 65 seconds in the aforementioned computer. The converged height and radiance functions of the reconstructed surface without imposing a weak statistical constraint $(\gamma=0)$ are shown in Fig. 4 (top). A 3-D representation of the reconstructed surface is displayed
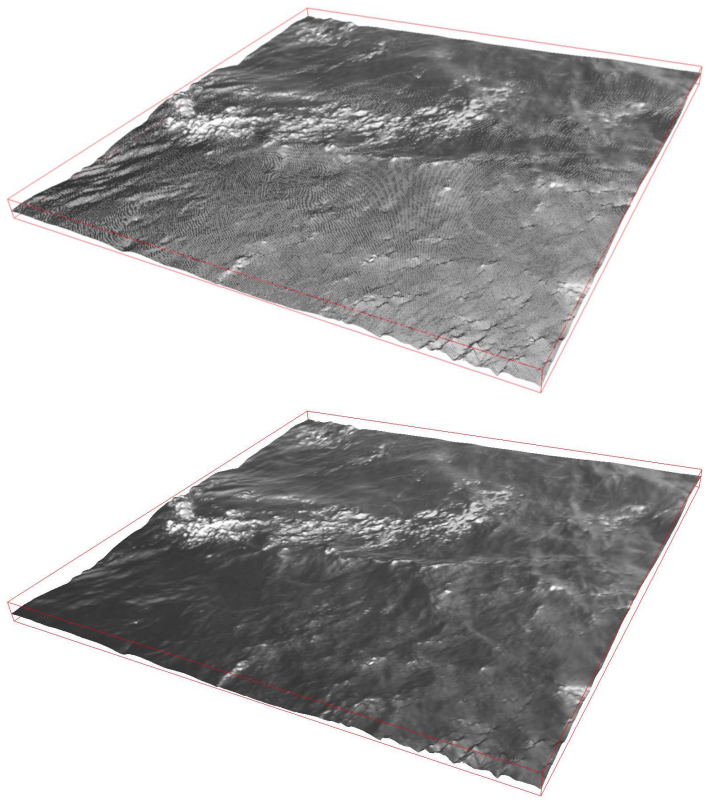

Fig. 5. Surface reconstruction without statistical constraint. Top: perspective 3-D wire-frame representation of the estimated surface shape (height) using a grid with $513^{2}$ points. Bottom: texture-mapped surface obtained by pasting the radiance function on the wire-frame model. The $Z$ axis has been expanded for visualization.

in Fig. 5. ${ }^{1}$ Both, $Z$ and $f$ are used to generate the modeled images in Fig. 4 (middle), which are a good fit of the observed images in Fig. 3. The absolute error images are also displayed, which better show the location of the discrepancies between modeled and original images.

Table II summarizes the different values of the cost terms obtained at the approximate solutions in this (1st column) and the next experiments. Fig. 6 shows the corresponding observed PDF using standardized height $\xi=\left(Z-\mu_{Z}\right) / \sigma_{Z}$ ( $\xi$ has zero mean and unit variance). Note the deviations from Gaussianity with large kurtosis. The associated omni-directional spectrum $\mathcal{S}(k)$ of $\xi$ is shown in Fig. 7 (dashed line). In a polar-reference

${ }^{1}$ A textured radiance (Fig. 4 top, left) on a smooth surface (Fig. 4 top, right) visually produces the effect of observing a rough surface (Fig. 5, bottom). This effect is exploited in computer graphics in the technique called "bump mapping" [23], where bumps and wrinkles on the surface of an object are simulated by perturbing the normal to the surface in lighting calculations ("radiance"), instead of perturbing the surface shape.

TABLE II

COST (ERROR) TERMS FOR DIFFERENT OPTIMIZATION STRATEGIES: WITH $(\gamma>0)$ OR WITHOUT $(\gamma=0)$ STATISTICAL CORRECTION AND WITH OR WITHOUT SIC PARAMETER ESTIMATION $\left(N_{s}=3\right)$.

\begin{tabular}{|l||c|c|c|c|}
\hline \multirow{2}{*}{} & \multicolumn{2}{|c|}{ No constraint, $\gamma=0$} & \multicolumn{2}{c|}{ CDF, $\gamma / A=1$} \\
\cline { 2 - 5 } & No SIC & SIC & No SIC & SIC \\
\hline \hline \multirow{2}{*}{$\#$ iters } & \multicolumn{2}{c|}{400} & \multicolumn{2}{c|}{600} \\
\hline$E_{\text {data }} / N_{p}$ & 37.82 & 13.53 & 37.99 & 13.68 \\
\hline$\alpha E_{\text {geom }} / N_{p}$ & 1.140 & 0.717 & 1.191 & 0.755 \\
\hline$\beta E_{\text {rad }} / N_{p}$ & 0.347 & 0.375 & 0.347 & 0.375 \\
\hline$\gamma \widetilde{E}_{\text {cdf }} / N_{p}$ & 0 & 0 & 0.015 & 0.012 \\
\hline$E / N_{p}$ (total) & 39.31 & 14.62 & 39.55 & 14.82 \\
\hline
\end{tabular}




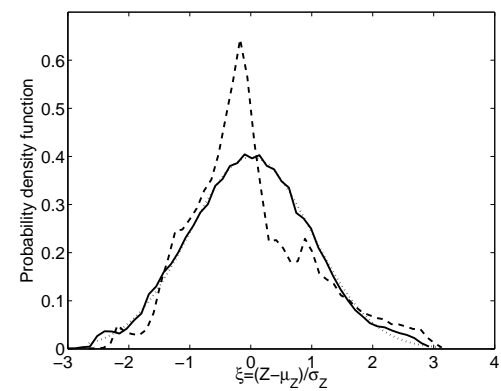

Fig. 6. Effect of the Statistical Constraint. Observed PDF of the reconstructed wave surface $Z$ with (solid line) and without (dashed line) statistical regularization. The normal distribution is plotted for comparison (dotted line).
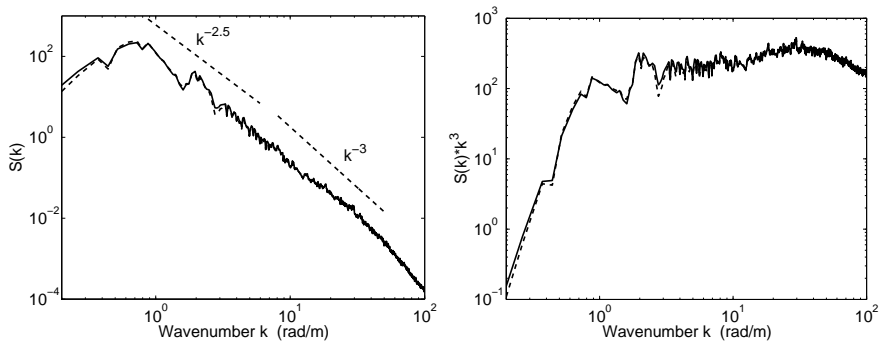

Fig. 7. Effect of the Statistical Constraint. Left: Omni-directional spectrum $\mathcal{S}(k)$ of the standardized reconstructed surface $\xi=\left(Z-\mu_{Z}\right) / \sigma_{Z}$ with (solid line) and without (dashed line) statistical regularizer. Right: Corresponding saturation spectra $k^{3} \mathcal{S}(k)$.

frame, $\mathcal{S}(k)$ is computed from the two-dimensional power spectrum $\Psi$ of $\xi$ as $\mathcal{S}(k)=\int_{0}^{2 \pi} \Psi(k, \theta) k d \theta$, where $k$ is the wavenumber and $\theta$ is the angle. The tail of the spectrum shows the decay rate change from $k^{-2.5}$ (inertial range, Zakharov spectrum [14]) to $k^{-3}$ (balanced dissipation range, Phillips' spectrum [24]).

To show the effect of the statistical constraint, 200 additional iterations (400 V-cycles) of multigrid are carried out using the cost augmented by (10), $\gamma \widetilde{E}_{\text {cdf }}$ with $\gamma / A=1$. This processing drives the surface toward the target distribution, Gaussian for simplicity although other distributions could have been used [15], [16]. This stage takes 50 seconds and decreases the statistical cost by roughly two orders of magnitude from $\gamma \widetilde{E}_{\text {cdf }} / N_{p}=1.765$ to 0.015 (3rd column of Table II) at the expense of slightly increasing the data fidelity and geometric cost terms (cf. 1st and 3rd columns of Table II). There are subtle differences between the height functions obtained with and without the statistical constraint (Figs. 8 and 4, respectively). Both solutions correctly capture the wave front moving toward the camera. Now, two non-linear terms (photometric fidelity and statistical constraint) compete to evolve the surface. The regions that change the most due to the statistical regularizer (see Fig. 9) are those with small photometric error (7), which are regions with small $\phi_{i}$ (e.g., smooth texture) and/or regions having a small footprint in the images (according to the Jacobian $\mathrm{J}_{i}$, points far away from the cameras have a smaller contribution to the data fidelity cost than points closer to the cameras). The statistical regularizer leaves the photometric error (Table II) and omni-directional spectrum (Fig. 7, right) almost unchanged while significantly modifying the PDF of the height map. This change is less pronounced if the statistical
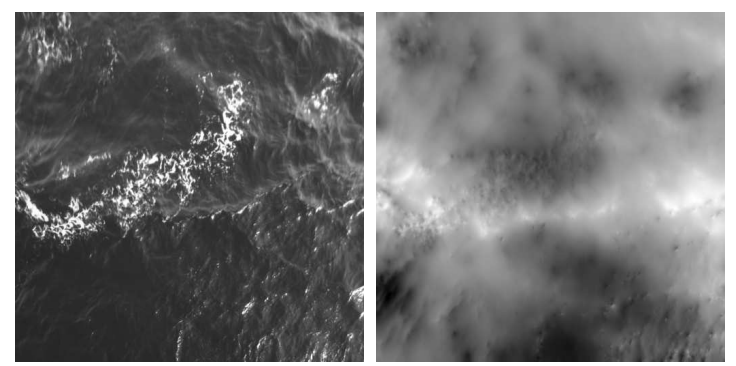

Fig. 8. Effect of the Statistical Constraint. Radiance and height functions after 200 iterations of multigrid incorporating the CDF statistical constraint.

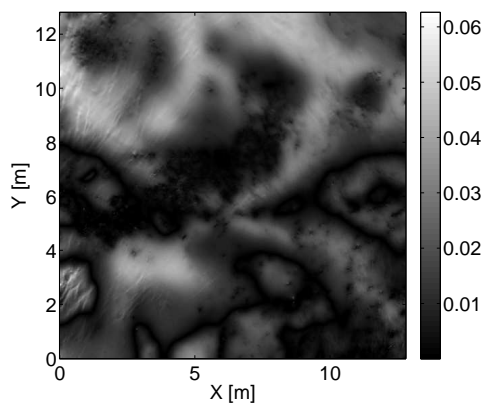

Fig. 9. Effect of the Statistical Constraint. Absolute difference of height functions (in meters): with and without statistical constraint. Bright values note the regions where the height function changed the most due to the statistical regularizer.

constraint is enforced on multiple consecutive snapshots, as it will be shown in section III-D. The new reconstructed surface is quasi-Gaussian as clearly shown in Fig. 6.

\section{B. Effect of the Photometric Compensation (SIC)}

Next, the steps of the previous experiment are repeated, but now enabling the estimation of the SIC parameters to reduce the photometric mismatch between generated and observed images. The results are summarized in columns 2 and 4 of Table II: the data fidelity error reduces significantly in case of enabling the compensation model (compare columns 1 vs 2 and 3 vs 4). Fig. 10 shows the reconstructed surface (height and radiance) for column 4 of Table II as well as the corresponding modeled and error images. The boundary of $\Omega_{i}$, which separates the modeled and original intensities in the modeled images, is more difficult to discern in case of using the SIC model (Fig. 10) than in the contrary (Fig. 4), for example near the left boundary of $\Omega_{i}$. This is illustrated in Fig. 11, which shows close-ups of the modeled images; the boundary of $\Omega_{1}$ can be identified by inspection of the foam patterns in Figs. 4 and 10 or by noticing that the boundary separates a slightly blurred region (modeled intensities, on the right of each image in Fig. 11) from the rest, a sharp region with the intensities of the original image. In addition, the effect of the photometric compensation is also noticeable in the smaller magnitude of the error images.

The linear photometric compensation model $\left(N_{s}=3\right.$ SIC parameters) in (25) offers a good compromise between numerical complexity and performance of the model, $\hat{I}_{i}=$ $a_{i} f\left(\mathbf{x}_{i}\right)+\theta_{i, 1}+\theta_{i, 2} x_{i}+\theta_{i, 3} y_{i}$. In experiments carried out, 


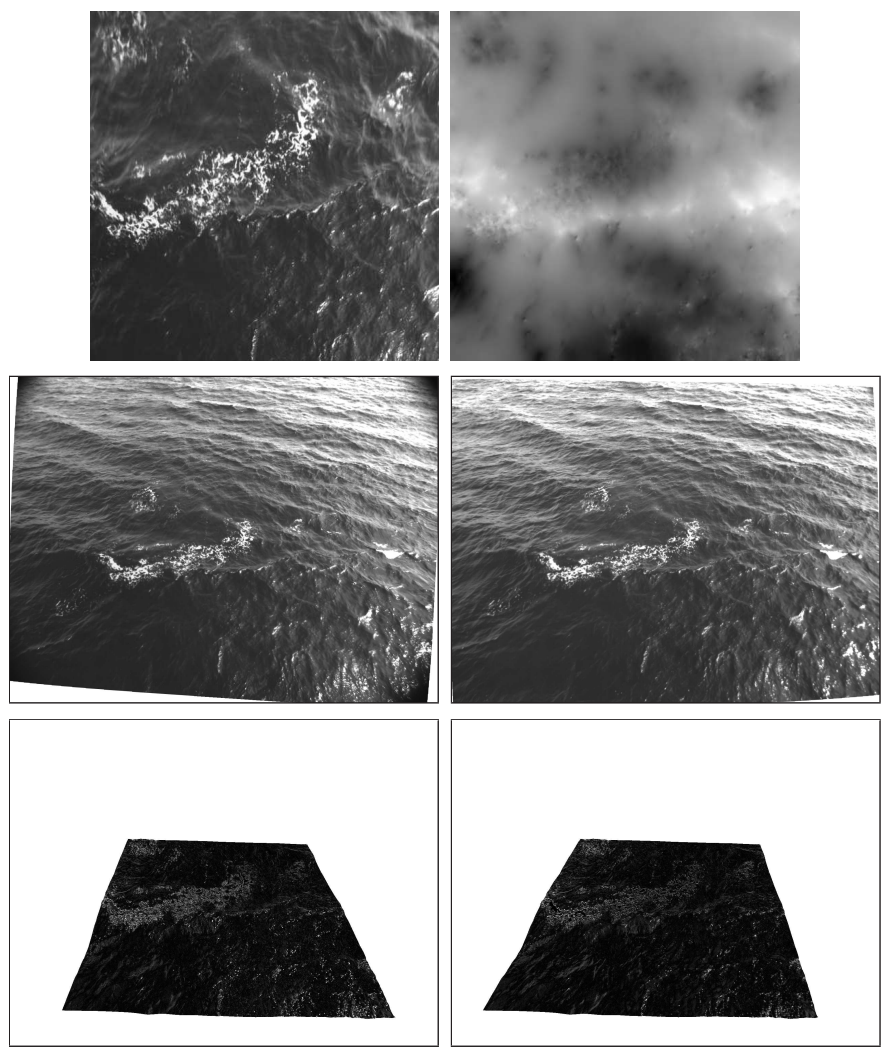

Fig. 10. Effect of the Photometric Compensation. Top: radiance and height functions, $f(\mathbf{u})$ and $Z(\mathbf{u})$, corresponding to column 4 of Table II. Minimization with CDF statistical constraint and SIC model with $N_{s}=3$ parameters. Middle: modeled images $\hat{I}_{i}, i=1,2$, superimposed on original images. Bottom: corresponding absolute error images $\left|I_{i}-\hat{I}_{i}\right|$ within the projection domains $\Omega_{i}$; error intensities have been amplified by a factor of 10 for visualization.
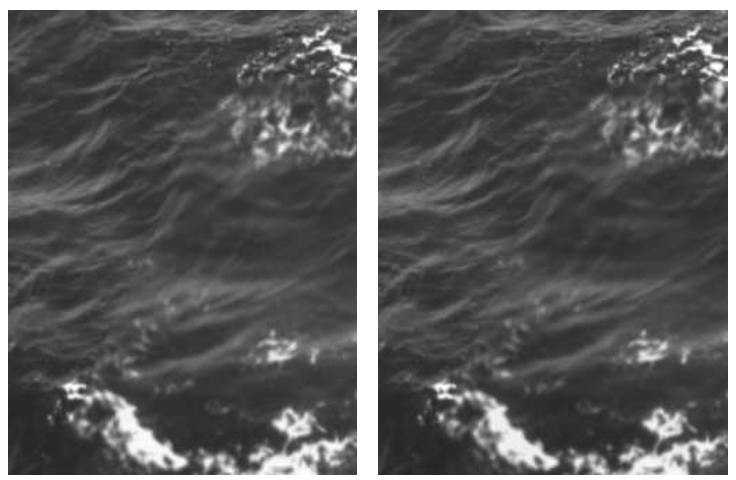

Fig. 11. Effect of the Photometric Compensation. Detail of the modeled image 1 in Figs. 4 and 10, respectively, near the top-left corner of $\Omega_{1}$. The boundary of $\Omega_{1}$ (i.e., the transition between the modeled and the original image) is less visible in the latter case due to the photometric compensation.

there is a big improvement from the cases $N_{s}=\{0,1\}$ to the case $N_{s}=3$, but the fit does not significantly improve when jumping to $N_{s}=6$ parameters.

The SIC model reduces shape artifacts in the regions corresponding to larger systematic intensity mismatches (cf. Figs. 8 and 10 near the corners). The PDF and omni-directional spectrum corresponding to the height function in Fig. 10 are plotted in Fig. 12; the results are very similar to those

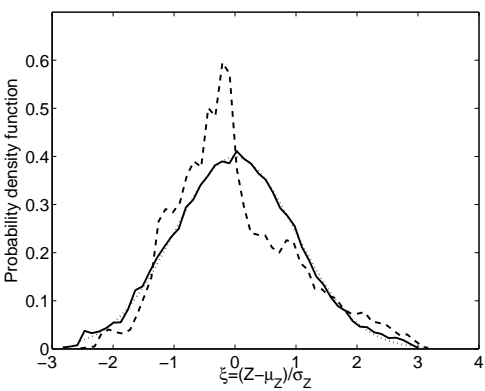

Fig. 12. Effect of the Photometric Compensation. SIC model with $N_{s}=3$ parameters. Observed PDF of the reconstructed wave surface $Z$ with (solid line) and without (dashed line) statistical regularization. The normal distribution is plotted for comparison (dotted line).
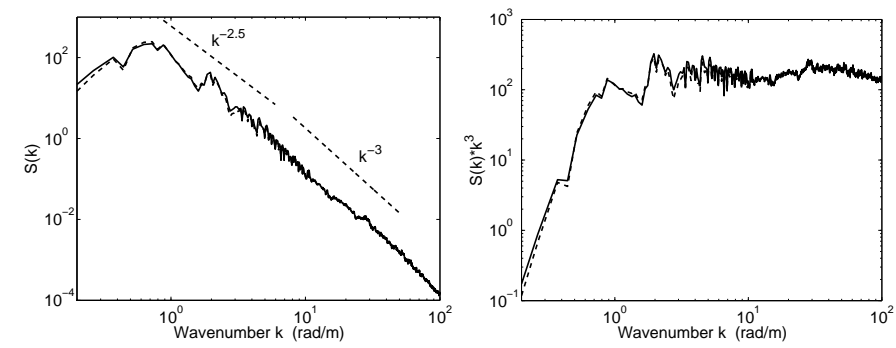

Fig. 13. Effect of the Photometric Compensation. SIC model with $N_{s}=3$ parameters. Left: Omni-directional spectrum $S(k)$ of the standardized reconstructed surface $\xi=\left(Z-\mu_{Z}\right) / \sigma_{Z}$ with (solid line) and without (dashed line) SIC model. Right: Corresponding saturation spectra $k^{3} \mathcal{S}(k)$.

in Fig. 6. The omni-directional spectra corresponding to the height functions of columns 2 and 4 of Table II are compared in Fig. 12: the SIC model attenuates high frequency artifacts. Due to the usefulness of the SIC model, it is enabled in the remaining experiments.

\section{Comparison of Statistical Penalties}

This section compares the effect of the four statistical penalties presented: CDF, Anderson-Darling (AD), Cramérvon Mises (CvM) and PDF (19). But first, some remarks about a careful implementation are appropriate. Empirical CDFs and PDFs have been calculated from histograms with $N_{b}=50$ bins. CDF values are linearly interpolated between samples, whereas PDF values are interpolated by splines. The PDF approach (19) requires the estimation of the derivative of the target and empirical PDFs (20). Moreover, the calculation of the time step $\Delta t$ and/or the linearization of the PDE also require the calculation of the second derivative of the PDF. Numerical derivatives of histograms have a noise amplification effect that can break down the statistical optimization. Therefore, filtering is essential to compute a smooth approximation of the derivatives of the PDF. The first derivative is the central part of the convolution of the PDF with the smooth derivative filter $d_{g}[n]=d[n] * w_{g}[n]$, where $d[n]=[1,0,-1] /(2 B)$ is the central difference filter corresponding to a bin size $B$ and $w_{g}[n]$ is a Gaussian smoothing filter. We chose $w_{g}[n]$ with $N_{b} / 10=5$ samples. The second derivative is the convolution of the first derivative with filter $d_{g}[n]$. Values between samples of the derivatives of the PDF are linearly interpolated. 
TABLE III

COST (ERROR) TERMS WITH SIC PARAMETER ESTIMATION $\left(N_{s}=3\right)$ FOR DIFFERENT STATISTICAL OPTIMIZATION STRATEGIES.

\begin{tabular}{|l||c|c|c|c|c|}
\hline & $\gamma=0$ & CDF & AD & CvM & PDF \\
\hline \hline$\#$ iters & 400 & \multicolumn{4}{|c|}{600} \\
\hline$\gamma / A$ & 0 & 1 & 0.45 & 6 & 0.5 \\
\hline$E_{\text {data }} / N_{p}$ & 13.53 & 13.68 & 13.65 & 13.80 & 13.64 \\
\hline$\alpha E_{\text {geom }} / N_{p}$ & 0.717 & 0.755 & 0.752 & 0.765 & 0.757 \\
\hline$\beta E_{\text {rad }} / N_{p}$ & 0.375 & 0.375 & 0.375 & 0.374 & 0.375 \\
\hline$\gamma \widetilde{E}_{\text {stat }} / N_{p}$ & 0 & 0.012 & 0.013 & 0.009 & 0.025 \\
\hline$E / N_{p}$ (total) & 14.62 & 14.82 & 14.79 & 14.95 & 14.80 \\
\hline
\end{tabular}
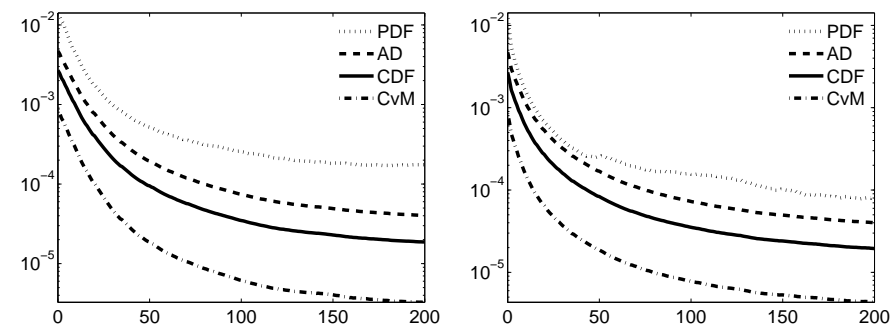

Fig. 14. Comparison of Statistical Penalties. Evolution of 4 statistical costs as the CDF approach (left) or the PDF approach (right) proceeds. Horizontal axis is the iteration counter. Same notation as in Fig. 2 for AD, CDF and CvM weights.

Table III compares all four statistical penalties. The relation between the values of $\gamma$ chosen for the CDF, AD and CvM penalties is determined by the approximate area under the weight functions (Table I and Fig. 2) in the interval $|\xi| \leq 3$. All four penalties successfully achieve the weak enforcement of the statistical constraint at the expense of increasing other terms in the composite cost functional.

Fig. 14 shows the evolution of all four costs as the CDF or PDF penalty iteration proceeds. As we can see, all four costs are correlated: the minimization of the CDF penalty implies the minimization of the other penalties and vice versa. The iterative solver decreases the costs by two orders of magnitude, with a steeper slope at the beginning than at the end: minimization is less effective as the iteration proceeds. The ordering of the weights in Table I, (Fig. 2) implies the same ordering of the costs: $\widetilde{E}_{\mathrm{CvM}} \leq \widetilde{E}_{\mathrm{cdf}} \leq \widetilde{E}_{\mathrm{AD}}$.

The deviations of the empirical PDFs obtained by all four statistical penalties with respect to the target distribution are displayed in Fig. 15 (the initial and the final empirical PDFs according to the CDF penalty are those in Fig. 12). Deviations are small in all cases, but a more detailed analysis shows the expected behaviour. Since the AD weight penalizes more the errors in the tails of the Gaussian (Fig. 2), the deviations are smaller in such regions away from $\xi=0$. The CvM weight does the opposite: it penalizes more the errors near $\xi=0$, and so, such deviations are smaller than those away from $\xi=0$. The unit weight (CDF) has an intermediate behaviour: deviations are equally distributed in $\xi$. The PDF penalty seems to give the smallest deviations among all four.

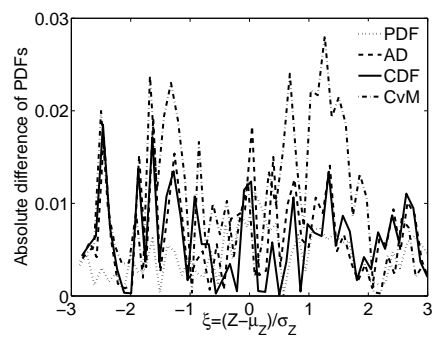

Fig. 15. Comparison of Statistical Penalties. Error between target and empirical standardized PDFs.

\section{Weak statistical constraints applied to the simultaneous reconstruction of multiple snapshots}

The variational stereo method developed can be naturally extended in several ways to process sequences of stereo images to generate a coherent space-time reconstruction of ocean waves. One of such methods is the manifold reconstruction (MR) presented in [20]. Moreover, the weak statistical technique can be combined with the MR method to enforce the physical properties of the waves in the entire space-time domain, which is closer to the spirit of statistics about using datasets richer than a single snapshot. This is illustrated in the next experiment that simultaneously reconstructs 1025 stereo snapshots acquired by the cameras at a $10 \mathrm{~Hz}$ frame rate. To make the computations feasible, the dataset is decimated by 4 in each spatial dimension: thus images are of size $406 \times 309$ pixels and a 3 -D grid with $129 \times 129 \times 1025$ points is chosen. To cover the same physical area, the spatial grid step is now $h=10 \mathrm{~cm}$. A 5-level FMG method with 1000 iterations per level is performed. Afterwards, 400 iterations of multigrid with statistical constraint (CDF penalty) are carried out. Such constraint is not enforced per snapshot, but on the entire space-time reconstructed surface. Fig. 16 shows two slices of the reconstructed height and radiance functions, which capture the oscillating patterns of the ocean waves. Fig. 17 displays the PDF of the height function before and after the weak enforcement of the statistical constraint, and the evolution of the statistical cost as the CDF iteration proceeds.
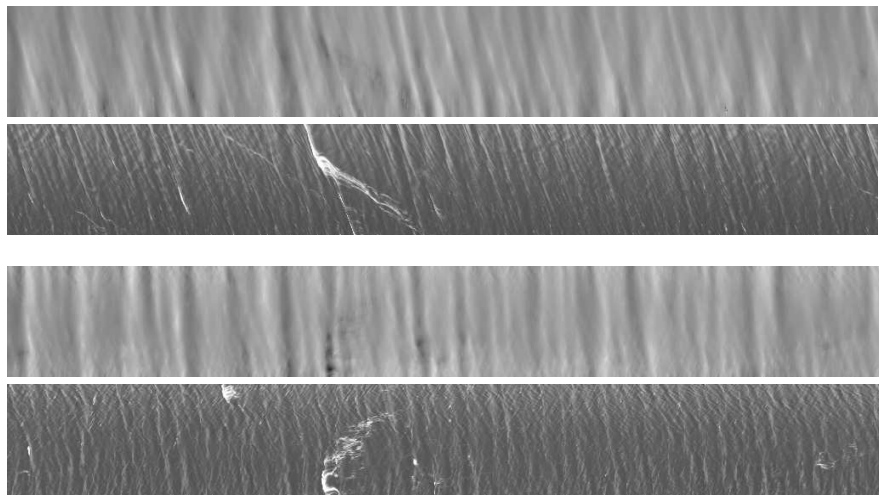

Fig. 16. Simultaneous reconstruction of multiple snapshots. Top: A slice at constant $u=u_{0}$. Surface height $Z\left(u_{0}, v, \tau\right)$ (grayscale encoded, from dark (low) to white (high)) and surface radiance $f\left(u_{0}, v, \tau\right)$. Horizontal axis is time $\tau$. Bottom: A slice at constant $v=v_{0}$ : height $Z\left(u, v_{0}, \tau\right)$ and radiance $f\left(u, v_{0}, \tau\right)$. 


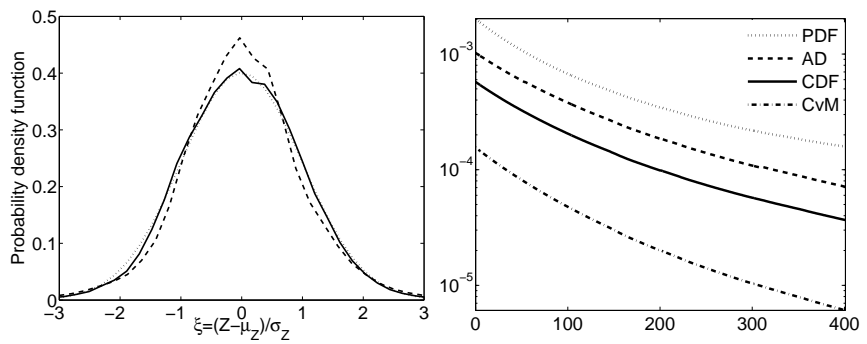

Fig. 17. Simultaneous reconstruction of multiple snapshots. Left: PDF of the height function with (solid line) and without (dashed line) statistical constraint. Right: evolution of 4 statistical costs during the 400 iterations that reduce the CDF penalty.
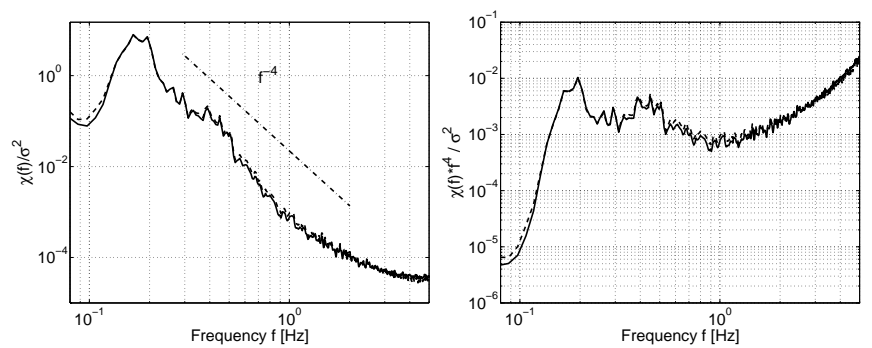

Fig. 18. Simultaneous reconstruction of multiple snapshots. Frequency spectrum. Left: Frequency spectrum of the standardized height time series with (solid line) and without (dashed line) statistical constraint. Right: Corresponding saturation spectra. The Nyquist frequency (half of the sampling frequency) is $5 \mathrm{~Hz}$, according to the snapshot (e.g. frame) rate.

The initial distribution computed over the entire 3-D grid is closer to Gaussianity than that of a single snapshot (previous experiments) due to the added temporal coherence between elevation maps of neighboring snapshots, and also in part due to the spatial decimation of the dataset. Comparing Figs. 14 and 17 , the spatial and temporal coupling of grid points seems to slightly decrease the convergence rate of the optimization of the functional.

Fig. 18 shows the results of a time series analysis on the reconstructed surface height $Z(u, v, \tau)$ analogous to that in [18]. The experiment proves the applicability of the developed technique for a relatively small observation time interval (102.5 s) from the point of view of ocean waves. Still, the spectrum $\chi(f)$ approximately shows an envelope with two peaks at the observed frequencies of $0.2 \mathrm{~Hz}$ (swell) and $0.4 \mathrm{~Hz}$ (wind waves), which agree well with those measured by other instrumentation in the platform. These are more visible in the saturation spectrum $\chi(f) f^{4}$ in Fig. 18 (right). The tail of the spectrum decays $\propto f^{-4}$, in agreement with the $k^{-2.5}$ decay rate of the omni-directional spectrum under the assumption of the dispersion relation $k=(2 \pi f)^{2} / g$ in deep-water, where $g$ is gravity acceleration.

\section{CONCLUSION}

Building upon the benefits of variational techniques and a stereo reconstruction method for smooth surfaces representable in the form of a graph supporting a smooth radiance function, we have shown how global properties of the object surface (ocean waves), such as statistical distributions, can be incorporated in the variational reconstruction framework via a weak constraint. We investigated four different expressions to measure the distance between the empirical distribution and the target model. We also discussed a spatial intensity compensation (SIC) and gain correction model to mitigate systematic intensity errors that may be present in the images. In addition, to overcome the weaknesses of the Lambertian generative model of the images in presence of specularities and/or abrupt intensity changes caused by sea foam, a robust photometric matching criterion (logarithmic image preprocessing) has been discussed. We successfully applied this method to reconstruct and enforce constraints on a small region of the surface of the ocean, both for a fixed time and for multiple snapshots (MR method). A thorough evaluation of the weak statistical constraint and the SIC model developed has been given both separately and together. In future research we plan to investigate new cost terms to incorporate more global and/or local properties of the dynamics of the object surface such as the wave equation, etc. Preliminary research shows that the developed image processing and remote sensing technology has a broad impact on ocean engineering since it allows to contrast and/or combine the understanding of oceanic sea states via statistical models with experimental measurements, enabling improved designs of off-shore structures and platforms.

\section{APPENDIX A}

\section{CALCULATION OF OBSERVED AND MODELED INTENSITIES}

Assuming that the surface $S$ is represented as a graph $Z=$ $Z(u, v)$, a point on the surface has coordinates

$$
\mathbf{X}(u, v)=(u, v, Z(u, v))^{\top} .
$$

The chain of operations to obtain the intensity $I_{i}\left(\mathbf{x}_{i}\right)$ corresponding to a surface point with world coordinates $\mathbf{X}(\mathbf{u}) \equiv$ $S(\mathbf{u}), \mathbf{u}=(u, v)^{\top}$, is

$$
\mathbf{X}(\mathbf{u}) \mapsto \tilde{\mathbf{X}}_{i}=\mathrm{M}^{i} \mathbf{X}+\mathbf{p}_{4}^{i} \mapsto \mathbf{x}_{i} \mapsto I_{i}\left(\mathbf{x}_{i}\right),
$$

where $\tilde{\mathbf{X}}^{i}=\left(\tilde{X}_{i}, \tilde{Y}_{i}, \tilde{Z}_{i}\right)^{\top}$ are related to the coordinates of $\mathbf{X}$ in the $i$-th camera frame, $\mathbf{x}_{i}=\left(\tilde{X}_{i} / \tilde{Z}_{i}, \tilde{Y}_{i} / \tilde{Z}_{i}\right)^{\top}$ is the projection of $\mathbf{X}$ in the $i$-th image plane and $\mathrm{P}^{i}=\left[\mathrm{M}^{i} \mid \mathbf{p}_{4}^{i}\right]$, with $\mathrm{M}^{i}=\mathrm{K}^{i} \mathrm{R}^{i} \equiv\left(\mathbf{n}_{1}^{i}, \mathbf{n}_{2}^{i}, \mathbf{n}_{3}^{i}\right)^{\top}$ and $\mathbf{p}_{4}^{i}=\mathrm{K}^{i} \mathbf{t}^{i}$. Also, $\left|\mathrm{M}^{i}\right|=$ $\operatorname{det}\left(\mathrm{M}^{i}\right)$.

The modeled intensity $\hat{I}_{i}$ depends on the radiance function $\hat{f}$ defined on the surface $S$, the point (e.g. pixel) location $\mathbf{x}_{i} \in \Omega_{i}$ and the SIC parameter vector $\boldsymbol{\vartheta}_{i}=\left(a_{i}, \boldsymbol{\theta}_{i}^{\top}\right)^{\top}$ for image $i$. More precisely, let us consider a photometric compensation model with a small number $N_{s}$ of parameters $\boldsymbol{\theta}_{i}=\left(\theta_{i, 1}, \ldots, \theta_{i, N_{s}}\right)^{\top}$ that describe a low spatial intensity compensation function $q_{i}\left(\mathbf{x}_{i}\right)=\mathbf{m}\left(\mathbf{x}_{i}-\mathbf{x}_{c}\right)^{\top} \boldsymbol{\theta}_{i}$, where $\mathbf{m}\left(\mathbf{x}_{i}\right)^{\top}=\left\{(1),\left(1, x_{i}, y_{i}\right),\left(1, x_{i}, y_{i}, x_{i}^{2}, x_{i} y_{i}, y_{i}^{2}\right)\right\}$ for $N_{s}=$ $\{1,3,6\}$, respectively. The center of the image is a sensible choice for $\mathbf{x}_{c}$ (the origin of the axes for the intensity function $q_{i}\left(\mathbf{x}_{i}\right)$ ), although other values are possible. The modeled intensity is

$$
\hat{I}_{i}\left(\mathbf{x}_{i}, f, \boldsymbol{\vartheta}_{i}\right)=a_{i} f\left(\mathbf{x}_{i}\right)+q_{i}\left(\mathbf{x}_{i}\right),
$$

with $f$ in (25) naturally defined by $f\left(\mathbf{x}_{i}\right):=\hat{f}\left(\pi_{i}^{-1}\left(\mathbf{x}_{i}\right)\right)$, where $\pi_{i}^{-1}$ stands for the back-projection operation from a 
point in the $i$-th image to the closest surface point with respect to the camera (see Fig. 1). By abusing notation, we use $f$ in (6) to write the parametrized radiance $f(\mathbf{u})$, understanding that $f\left(\mathbf{x}_{i}\right)$ in (25) reads the back-projected value in $\hat{f}(\mathbf{X}(\mathbf{u}))=$ $f(\mathbf{u})$. Observe that if $\boldsymbol{\vartheta}_{i}=\left(1, \mathbf{0}^{\top}\right)^{\top}$ there is no automatic gain control or spatial intensity compensation, $\hat{I}_{i}=f\left(\mathbf{x}_{i}\right)$.

\section{APPENDIX B}

\section{JACOBIAN BETWEEN INTEGRATION DOMAINS $\Omega_{i}$ AND $U$}

The Jacobian of the change of variables between integration domains $\Omega_{i}$ and $U$ is, by applying the chain rule to (24),

$$
\mathrm{J}_{i}=\left|\operatorname{det}\left(\frac{d \mathbf{x}_{i}}{d \mathbf{u}}\right)\right|=-\left|\mathrm{M}^{i}\right| \tilde{Z}_{i}^{-3}\left(\mathbf{X}-\mathbf{C}_{i}\right) \cdot\left(\mathbf{X}_{u} \times \mathbf{X}_{v}\right),
$$

where $\mathbf{X}_{u} \times \mathbf{X}_{v}$ is proportional to the outward unit normal $\mathbf{N}$ to the surface at $\mathbf{X}(u, v)$, and $\tilde{Z}_{i}=\mathbf{n}_{3}^{i} \cdot\left(\mathbf{X}-\mathbf{C}_{i}\right)>0$ is the depth of the point $\mathbf{X}$ with respect to the $i$-th camera (located at $\mathbf{C}_{i}$ ). Observe that the Jacobian weights the photometric error $\phi_{i}$ in (7) proportionally to the cosine of the angle between the unit normal to the surface at $\mathbf{X}$ and the projection ray $\mathbf{X}-\mathbf{C}_{i}$ (the ray joining the optical center of the camera and $\mathbf{X}$ ).

\section{APPENDIX C}

OPTIMALITY OF THE FUNCTIONAL WITH RESPECT TO THE SPATIAL INTENSITY COMPENSATION (SIC) PARAMETERS

The optimality condition (12) yields a linear system of equations $\mathrm{A} \boldsymbol{\vartheta}=\mathbf{b}$ in all SIC parameters. However, since $\partial E_{i} / \partial \boldsymbol{\vartheta}_{k}=\mathbf{0}$ if $i \neq k$, matrix $\mathrm{A}$ is block diagonal and the system splits into one linear system for each image, $\mathrm{A}_{i} \boldsymbol{\vartheta}_{i}=\mathbf{b}_{i}$, with $\mathrm{A}_{i}=\int_{\Omega_{i}} \mathbf{w}_{i}\left(\mathbf{x}_{i}\right) \mathbf{w}_{i}\left(\mathbf{x}_{i}\right)^{\top} d \mathbf{x}_{i}, \mathbf{b}_{i}=\int_{\Omega_{i}} I_{i}\left(\mathbf{x}_{i}\right) \mathbf{w}_{i}\left(\mathbf{x}_{i}\right) d \mathbf{x}_{i}$, and $\mathbf{w}_{i}\left(\mathbf{x}_{i}\right)=\left(f\left(\mathbf{x}_{i}\right), \mathbf{m}\left(\mathbf{x}_{i}\right)^{\top}\right)^{\top}$. Matrices $\mathrm{A}_{i}$ are square and symmetric. The model contains $\left(N_{s}+1\right)$ SIC parameters per image. However, since no constraints on $f$ are imposed, the model is over-parametrized and multiple solutions may exist. To address this problem, a reference image is chosen, e.g. the first one, with $\boldsymbol{\vartheta}_{1}=\left(1, \mathbf{0}^{\top}\right)^{\top}$. Therefore, the number of SIC parameters in the model is $\left(N_{s}+1\right)\left(N_{c}-1\right)$. In the nested iterative scheme, all $\mathrm{A}_{i}$ and $\mathbf{b}_{i}$ should be re-computed in every outer iteration for $N_{s} \geq 3$ since they depend on $Z$.

\section{APPENDIX D}

\section{STATISTICAL CONSTRAINT. FIRST VARIATION}

Since the statistical penalty does not affect the boundary condition (14), to compute the first variation of (10) we can either use the definition of the Gâteaux derivative or augment $Z$ with an artificial variable $t$, i.e., $Z=Z(\mathbf{u}, t)$, so that the functional depends on $t$, differentiate with respect to this variable and exploit the relationship between both derivatives:

$$
\frac{d}{d t} E(Z)=\int_{U} \frac{\delta E}{\delta Z} Z_{t} d \mathbf{u} \doteq\left\langle\frac{\delta E}{\delta Z}, Z_{t}\right\rangle_{L^{2}(U)} .
$$

In (10), carrying out operations in the distributional sense,

$$
\begin{aligned}
\frac{d E_{\mathrm{cdf}}}{d t} & =\int_{-\infty}^{\infty}\left(G(z)-\operatorname{cdf}^{Z}(z)\right) \frac{1}{A} \int_{U} \delta(z-Z(\mathbf{u})) Z_{t} d \mathbf{u} d z \\
& =\int_{U} \frac{1}{A} \int_{-\infty}^{\infty}\left(G(z)-\operatorname{cdf}^{Z}(z)\right) \delta(z-Z(\mathbf{u})) d z Z_{t} d \mathbf{u} \\
& =\left.\int_{U} \frac{1}{A}\left(G(z)-\operatorname{cdf}^{Z}(z)\right)\right|_{z=Z(\mathbf{u})} Z_{t} d \mathbf{u} \\
& =\int_{U} \frac{\delta E_{\mathrm{cdf}}}{\delta Z} Z_{t} d \mathbf{u},
\end{aligned}
$$

where the functional derivative of $E_{\mathrm{cdf}}$ with respect to $Z$ is

$$
\frac{\delta E_{\mathrm{cdf}}}{\delta Z}(Z(\mathbf{u}))=\left.\frac{1}{A}\left(G(z)-\operatorname{cdf}^{Z}(z)\right)\right|_{z=Z(\mathbf{u})} .
$$

In the PDF-based statistical penalty (19), we have

$$
\begin{aligned}
\frac{d E_{\mathrm{pdf}}}{d t} & =\int_{-\infty}^{\infty}\left(G^{\prime}(z)-\mathrm{pdf}^{Z}(z)\right) \frac{1}{A} \int_{U} \delta^{\prime}(z-Z(\mathbf{u})) Z_{t} d \mathbf{u} d z \\
& =\int_{U} \frac{1}{A} \int_{-\infty}^{\infty}\left(G^{\prime}(z)-\operatorname{pdf}^{Z}(z)\right) \delta^{\prime}(z-Z(\mathbf{u})) d z Z_{t} d \mathbf{u} \\
& =\left.\int_{U} \frac{1}{A}(-1) \frac{d}{d z}\left(G^{\prime}(z)-\operatorname{pdf}^{Z}(z)\right)\right|_{z=Z(\mathbf{u})} Z_{t} d \mathbf{u} \\
& =\int_{U} \frac{\delta E_{\mathrm{pdf}}}{\delta Z} Z_{t} d \mathbf{u},
\end{aligned}
$$

where we used the rule for the $n$-derivative of the Dirac $\delta$ distribution:

$$
\int_{x-a}^{x+a} f(t) \delta^{(n)}(x-t) d t=(-1)^{n} f^{(n)}(x) .
$$

Hence, the functional derivative of $E_{\mathrm{pdf}}$ with respect to $Z$ is

$$
\frac{\delta E_{\mathrm{pdf}}}{\delta Z}(Z(\mathbf{u}))=-\left.\frac{1}{A} \frac{d}{d z}\left(G^{\prime}(z)-\operatorname{pdf}^{Z}(z)\right)\right|_{z=Z(\mathbf{u})} .
$$

\section{APPENDIX E}

\section{FIRST VARIATION OF THE STATISTICAL CONSTRAINT WITH VARYING $\sigma_{Z}$}

What are the functional derivatives of $\widetilde{E}_{\mathrm{cdf}}=\sigma_{Z}^{-1} E_{\mathrm{cdf}}$ in (10) and $\widetilde{E}_{\mathrm{pdf}}=\sigma_{Z} E_{\mathrm{pdf}}$ in (19) assuming a varying $\sigma_{Z}$ ? To address these questions, we use two important results.

Result 1: The expected value $E[\cdot]$ of a function $q(Z)$ of the empirical height $Z$ is

$$
E[q(Z)]=\frac{1}{A} \int_{U} q(Z(\mathbf{u})) d \mathbf{u}
$$

The proof is the following:

$$
\begin{aligned}
E[q(Z)] & =\int_{-\infty}^{\infty} q(z) \operatorname{pdf}^{Z}(z) d z \\
& =\int_{-\infty}^{\infty} q(z) \frac{1}{A} \int_{U} \delta(z-Z(\mathbf{u})) d \mathbf{u} d z \\
& =\frac{1}{A} \int_{U} \int_{-\infty}^{\infty} q(z) \delta(z-Z(\mathbf{u})) d z d \mathbf{u} \\
& =\frac{1}{A} \int_{U} q(Z(\mathbf{u})) d \mathbf{u}
\end{aligned}
$$


Three interesting cases of (29) are the mean, the variance and the characteristic function. The interpretation of the mean is simple: it is the average height over $U$,

$$
\mu_{Z}=E[Z]=\frac{1}{A} \int_{U} Z(\mathbf{u}) d \mathbf{u}
$$

The variance is the average height spread around the mean height value over the domain $U$,

$$
\sigma_{Z}^{2}=E\left[\left(Z-\mu_{Z}\right)^{2}\right]=\frac{1}{A} \int_{U}\left(Z(\mathbf{u})-\mu_{Z}\right)^{2} d \mathbf{u}
$$

The characteristic function is the Fourier transform of the PDF,

$$
\operatorname{Char}^{Z}(\omega)=E\left[e^{\imath \omega Z}\right]=\frac{1}{A} \int_{U} e^{\imath \omega Z(\mathbf{u})} d \mathbf{u}
$$

Result 2: The functional derivative of the expectation (29) of a function $q$ of the empirical height distribution is

$$
\frac{\delta}{\delta Z} E[q(Z)]=\frac{1}{A} q^{\prime}(Z(\mathbf{u})) .
$$

The proof based on Result 1 . Augment $Z$ with an artificial time variable, $Z=Z(\mathbf{u}, t)$, so that the expected value depends on $t$, differentiate with respect to this variable and exploit the relationship between both derivatives:

$$
\frac{d}{d t} E[q(Z)] \stackrel{(29)}{=} \frac{1}{A} \int_{U} q^{\prime}(Z(\mathbf{u})) Z_{t} d \mathbf{u}=\int_{U} \frac{\delta E[q(Z)]}{\delta Z} Z_{t} d \mathbf{u} .
$$

In particular, the functional derivatives of the mean and the standard deviation are, respectively, $\frac{\delta}{\delta z} \mu_{Z}=\frac{1}{A}$ and

$$
\begin{aligned}
\frac{\delta}{\delta Z} \sigma_{Z} & =\frac{\delta}{\delta Z} \sqrt{E\left[\left(Z-\mu_{Z}\right)^{2}\right]} \\
& =\frac{1}{2 \sigma_{Z}} \frac{\delta}{\delta Z} E\left[\left(Z-\mu_{Z}\right)^{2}\right] \\
& \stackrel{(31)}{=} \frac{1}{A} \frac{Z(\mathbf{u})-\mu_{Z}}{\sigma_{Z}} .
\end{aligned}
$$

Let us now answer the above questions. By the chain rule, using Leibniz's notation,

$$
\begin{aligned}
\frac{\delta \widetilde{E}_{\mathrm{cdf}}}{\delta Z} & =\frac{\delta}{\delta Z}\left(\sigma_{Z}^{-1} E_{\mathrm{cdf}}\right) \\
& =\sigma_{Z}^{-1} \frac{\delta E_{\mathrm{cdf}}}{\delta Z}-\frac{E_{\mathrm{cdf}}}{\sigma_{Z}^{2}} \frac{\delta \sigma_{Z}}{\delta Z} \\
& =\frac{1}{A \sigma_{Z}}\left(\left.\left(G(z)-\operatorname{cdf}^{Z}(z)\right)\right|_{z=Z}-\widetilde{E}_{\mathrm{cdf}} \frac{Z-\mu_{Z}}{\sigma_{Z}}\right),
\end{aligned}
$$

where we used (27) and (32).

Regarding the PDF approach, using (28) and (32),

$$
\begin{aligned}
\frac{\delta \widetilde{E}_{\mathrm{pdf}}}{\delta Z} & =\frac{\delta}{\delta Z}\left(\sigma_{Z} E_{\mathrm{pdf}}\right) \\
& =\sigma_{Z} \frac{\delta E_{\mathrm{pdf}}}{\delta Z}+E_{\mathrm{pdf}} \frac{\delta \sigma_{Z}}{\delta Z} \\
& =-\left.\frac{\sigma_{Z}}{A} \frac{d}{d z}\left(G^{\prime}(z)-\operatorname{pdf}^{Z}(z)\right)\right|_{z=Z}+\frac{\widetilde{E}_{\mathrm{pdf}}}{A \sigma_{Z}} \frac{\left(Z-\mu_{Z}\right)}{\sigma_{Z}} .
\end{aligned}
$$

\section{ACKNOWLEDGMENT}

Research supported by the Office of Naval Research Grant BAA 09-012: "Ocean Wave Dissipation and energy Balance (WAVE-DB): toward reliable spectra and first breaking statistics". G. Gallego is supported by the Marie Curie COFUND grant of the EU. This work has been partially supported by the Ministerio de Ciencia e Innovación of the Spanish Government under project TEC2010-20412 (Enhanced 3DTV).

\section{REFERENCES}

[1] K. Holland, R. Holman, T. Lippmann, J. Stanley, and N. Plant, "Practical use of video imagery in nearshore oceanographic field studies," Oceanic Engineering, IEEE Journal of, vol. 22, no. 1, pp. 81-92, jan. 1997.

[2] A. Benetazzo, "Measurements of short water waves using stereo matched image sequences," Coastal Engineering, vol. 53, no. 12, pp. 1013-1032, 2006.

[3] J. M. Wanek and C. H. Wu, "Automated trinocular stereo imaging system for three- dimensional surface wave measurements," Ocean Engineering, vol. 33, no. 5-6, pp. 723-747, 2006.

[4] G. Gallego, A. Benetazzo, A. Yezzi, and F. Fedele, "Wave Statistics and Spectra via a Variational Wave Acquisition Stereo System," in Proc. 27th Int. Conf. on Offshore Mechanics and Arctic Engineering (OMAE), Estoril, Portugal, June 2008, pp. 801-808, paper OMAE 2008-57160.

[5] A. J. Bechle and C. H. Wu, "Virtual wave gauges based upon stereo imaging for measuring surface wave characteristics," Coastal Engineering, vol. 58, no. 4, pp. 305-316, 2011.

[6] M. V. Kosnik and V. A. Dulov, "Extraction of short wind wave spectra from stereo images of the sea surface," Measurement Science and Technology, vol. 22, no. 1, p. 015504, 2011.

[7] S. de Vries, D. Hill, M. de Schipper, and M. Stive, "Remote sensing of surf zone waves using stereo imaging," Coastal Engineering, vol. 58, no. 3, pp. 239-250, 2011 .

[8] A. Benetazzo, F. Fedele, G. Gallego, P.-C. Shih, and A. Yezzi, "Offshore stereo measurements of gravity waves," Coastal Engineering, vol. 64, no. 0, pp. 127-138, 2012.

[9] F. Fedele, A. Benetazzo, G. Gallego, P.-C. Shih, A. Yezzi, F. Barbariol, and F. Ardhuin, "Space-time measurements of oceanic sea states," Ocean Modelling, 2013, doi:10.1016/j.ocemod.2013.01.001.

[10] Y. Ma, S. Soatto, J. Kosecka, and S. Sastry, An Invitation to $3 D$ Vision: From Images to Geometric Models. Springer Verlag, 2004.

[11] O. D. Faugeras and R. Keriven, "Variational principles, surface evolution, PDEs, level set methods, and the stereo problem," IEEE Trans. Image Proc., vol. 7, no. 3, pp. 336-344, 1998.

[12] A. Yezzi and S. Soatto, "Stereoscopic Segmentation," International Journal of Computer Vision, vol. 53, no. 1, pp. 31-43, 2003.

[13] M. S. Longuet-Higgins, "The effect of non-linearities on statistical distributions in the theory of sea waves," Journal of fluid mechanics, vol. 17, pp. 459-480, 1963.

[14] V. E. Zakharov, "Statistical theory of gravity and capillary waves on the surface of a finite-depth fluid," Eur. J. Mech. B - Fluids, vol. 18, no. 3, pp. 327-344, 1999.

[15] H. Socquet-Juglard, K. Dysthe, K. Trulsen, H. E. Krogstad, and J. Liu, "Probability distributions of surface gravity waves during spectral changes," Journal of Fluid Mechanics, vol. 542, pp. 195-216, 2005.

[16] A. Tayfun and F. Fedele, "Wave height distributions and nonlinear effects," Ocean Engineering, vol. 34, no. 11-12, pp. 1631,1649, 2007.

[17] G. Gallego, A. Yezzi, F. Fedele, and A. Benetazzo, "Weak Statistical Constraints for Variational Stereo Imaging of Oceanic Waves," in Third Int. Conf., SSVM 2011, ser. Lecture Notes in Computer Science, vol. 6667, 2012, pp. 520-531.

[18] — "A Variational Stereo Method for the Three-Dimensional Reconstruction of Ocean Waves," Geoscience and Remote Sensing, IEEE Trans., vol. 49, no. 11, pp. 4445-4457, Nov. 2011.

[19] R. I. Hartley and A. Zisserman, Multiple View Geometry in Computer Vision, 2nd ed. Cambridge University Press, 2004.

[20] G. Gallego, "Variational Image Processing Algorithms for the Stereoscopic Space-Time Reconstruction of Water Waves," Ph.D. dissertation, Georgia Institute of Technology, Atlanta, GA, USA, 2011, directors: Yezzi, A. and Fedele, F.

[21] T. Anderson and D. Darling, "A Test of Goodness-of-Fit," Journal of the American Statistical Association, vol. 49, pp. 765-769, 1954.

[22] W. L. Briggs, V. E. Henson, and S. F. McCormick, A Multigrid Tutorial, Second Edition. SIAM, 2000. 
[23] J. F. Blinn, "Simulation of wrinkled surfaces," in SIGGRAPH Comput. Graph., vol. 12, no. 3. New York, NY, USA: ACM, Aug. 1978, pp. 286-292.

[24] O. Phillips, "The equilibrium range in the spectrum of wind-generated waves," Journal of Fluid Mechanics, vol. 4, no. 4, pp. 426-434, 1958.

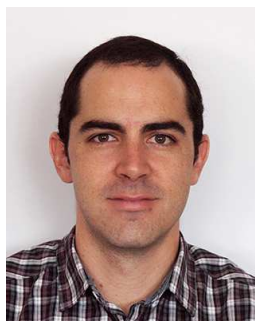

Guillermo Gallego received the Ingeniero de Telecomunicación degree (five-year engineering program) from Universidad Politécnica de Madrid, Madrid, Spain, in 2004, the M.S. degree in mathematical engineering (Magíster en Ingeniería Matemática) from Universidad Complutense de Madrid, Madrid, in 2005, and the M.S. in electrical and computer engineering, the M.S. in mathematics, and the Ph.D. in electrical and computer engineering from the Georgia Institute of Technology, Atlanta, GA, USA, in 2007, 2009 and 2011, respectively.

He was a recipient of the Fulbright Scholarship to pursue graduate studies at the Georgia Institute of Technology in 2005. Since 2011, he has been a MarieCurie COFUND post-doctoral researcher with the Universidad Politécnica de Madrid. His research interests fall within the areas of signal processing, geometry, optimization, computer vision and ocean engineering.

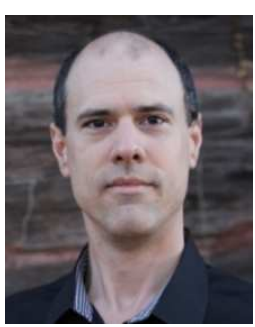

Anthony Yezzi was born in Gainsville, FL, USA. He obtained both his B.S. and Ph.D. degrees with minors in mathematics and music from the Department of Electrical Engineering, University of Minnesota, Minneapolis, MN, USA.

He continued his research as a Post-doctoral Research Associate with the Laboratory for Information and Decision Systems, Massachusetts Institute of Technology, Boston, MA, USA. Since the fall of 1999, he has been a faculty member with the Georgia Institute of Technology, Atlanta, GA, USA. His research interests fall broadly within the fields of image processing and computer vision. In particular he is interested in curve and surface evolution theory and partial differential equation techniques as they apply to topics within these fields (such as segmentation, image smoothing and enhancement optical flow, stereo disparity, shape from shading, object recognition, and visual tracking). Much of his work is particularly tailored to problems in medical imaging, including cardiac ultrasound, MRI, and computer tomography. $\mathrm{He}$ consults with industry in the areas of visual inspection and medical imaging.

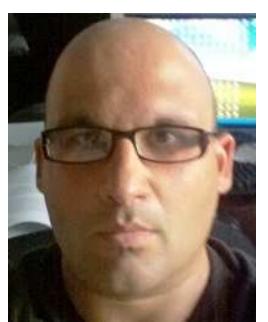

Francesco Fedele received the Laurea degree (magna cum laude) in civil engineering from the University Mediterranea, Reggio Calabria, Italy, in 1998, and Ph.D. degree in civil engineering from the University of Vermont, Burlington, VT, USA, in 2004.

$\mathrm{He}$ is an Associate Professor with the School of Civil and Environmental Engineering and the School of Electrical and Computer Engineering, Georgia Institute of Technology (Georgia Tech), Atlanta, GA, USA. He became a Faculty Member with Georgia Tech in 2007 after a post-doctoral research position at the NASA Goddard Space Flight Center, Greenbelt, MD, USA. His current research focus is on nonlinear wave phenomena, fluid mechanics, sustainable ocean energy, computational methods and inverse problems. The corresponding research thrusts include wave turbulence and rogue waves, algorithms for biomedical tomographic imaging, mathematical modeling, and experimentation on renewable devices to harness energy from tidal streams.

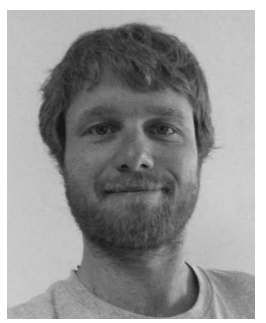

Alvise Benetazzo received the Laurea degree (magna cum laude) in civil engineering and the $\mathrm{Ph} . \mathrm{D}$. degree in civil and environmental engineering from the University of Padua, Padua, Italy, in 2000 and 2006, respectively.

$\mathrm{He}$ is a young Researcher with the Institute of Marine Science (ISMAR), National Research Council (CNR), Venice, Italy. Before joining ISMAR in 2011, he was a Project Manager with Protecno S.r.l., Padua. His expertise is in mathematical and physical models of water phenomena, measurements and analysis of water waves by using remote sensors, and remote sensing models for detecting illegal landfill. While at Protecno, he has been involved in all the aspects of both mathematical and physical modeling of the MOdulo Sperimentale Elettromeccanico (MOSE) structure at the Chioggia and Treporti inlets in Venice, Italy. 